\title{
13
}

\section{Promoting interdisciplinary and intercultural intentions through the history of informatics}

\author{
Klaus-D. Graf \\ Institut für Informatik, Freie Universität Berlin \\ Berlin, Germany
}

\begin{abstract}
The integration of informatics and information technology into schools should not only be oriented towards subject matter and methods but also towards general current demands of society on education. Interdisciplinary and intercultural knowledge and abilities form part of these demands. They can be promoted by aspects of the history of informatics such as early binary coding in China or early calculating machines emanating from roots in different cultures - Arabian, Chinese and European.
\end{abstract}

Keywords: culture, innovation, instruction, interdisciplinary, pedagogy

\section{INTRODUCTION}

The integration of new subject matter and methods in education at our schools is normally influenced by different intentions, very often contradictory, one more passive and one more active or even aggressive. The emphasis of the first lies in the support or the extension of existing matter and methods; the emphasis of the second lies in the introduction of new subject-matter and methods, partially accompanied by ousting 
traditional matter. Different opinions also occur about the character of innovative intentions. They can be strongly oriented towards the subject or strongly determined by the general expectations of society from education.

These very general statements relate directly to issues associated with the introduction of elements from informatics or information technology in education. The fundamental position of this paper is that the main task is not the extensive integration of informatics and information technology in education, but the improvement of education in general, as expected and demanded by a society faced with ever-growing problems of enormous complexity. Informatics will be of great importance for this task, and not just related to the more narrow objectives of its own.

\section{INFORMATION TECHNOLOGY AND SOCIETY}

Ruiz reports that decision makers in education agree that modern societies rely increasingly on larger numbers of individuals with high-level knowledge and skills at their disposal [1]. This means, in addition to elementary cultural techniques such as reading, writing and calculating, school has to teach other skills such as analysing, abstracting and modelling. Conceptual and communication skills are also required in the fields of production and service. An increasing range of jobs demand abilities and talents from individuals who know about their responsibilities in a wide context, and many. of these people must be able to work in multi-disciplinary teams.

Moreover, further high-level skills than those listed above are needed if one considers the challenges to societies by universal problems such as the world economy or environment. These problems are of extreme complexity and require a great amount of international interaction. Any success in these activities is dependent on mutual understanding and acknowledgement of different traditions and attitudes concerning elements of these problems, such as mass production, environmental protection, political and social structures. This understanding is required not only from a few experts at a high level, but from anyone engaged in problems related to foreign concerns. Readiness and goodwill have to be developed, so more attention has to be given to intercultural intentions in education. Teaching foreign languages is only a beginning; it must be accompanied by reflecting the relations between any two respective nations and their cultures in present time and in history. This in turn leads to a need to understand the influence of foreign culture on other cultures. These are demands on a very high level; it is well understood that in school only 
very elementary first steps can be taken. But they have to be taken there to ensure a modest contribution of our school education to solving the problems of our societies.

At the present time, there appear to be substantial problems in relationships between Arabia, China and the Western World. More mutual knowledge about the history of these cultures could be a small contribution to tackling these problems of global relationships in a peaceful way.

\section{THE ROLE OF INFORMATION TECHNOLOGY}

There are avenues in most subjects in school to pursue intentions such as the ones discussed above and every subject could make its contribution. In particular, informatics and information technology form a rich source for contributing to such mutual understanding.

Analysing, abstracting, modelling and conceptual skills are fundamental ideas in software engineering, and elementary techniques from this field can be integrated into informatics education. Communicational and inter-disciplinary skills are developed when solving problems by project teams. A feeling of responsibility for one's work can be developed when the consequences of informatics applications are considered systematically as a constituent part of the project and the project team. Also, intercultural awareness can be developed through studying the history of informatics; I will address this point further on in this paper.

\section{Didactical model}

I comment elsewhere on this practice [2]. Below is a representation of a didactical model for the teaching of informatics and its implications, which can enforce the consideration of intentions such as the ones related to responsibility, interdisciplinary cooperation or intercultural context as well as subject matter and methods of informatics. Instead of just putting a problem from informatics or information technology in the classroom and asking how to solve it, this problem should be regarded in a string of three questions. 


\section{WHERE FROM $\Rightarrow$ WHERE HERE AND NOW $\Rightarrow$ WHERE TO?}

- Where from?: Where does the problem come from? What are the causes or motives for it? Who is interested in the solution and who helps or opposes?

- Where here and now?: Which resources do we have here and now to solve the problem? How can it be done?

- Where to?: Where will the solution of the problem lead us to? Will we get what we want, and what else? What are the impacts and side effects in our environment, in society?

We can extend this scheme to questions to put to informatics and information technology in general.

Where does informatics come from? To answer this question we study the motives and causes of people who invented informatics tools or methods, we study the cultures or subcultures who were interested, we study the disciplines from which elements of informatics grew.

Where is informatics here and now? What is informatics? To answer this we study tools for description, analysis and design of information processing.

Where does informatics lead to? To answer this we study the social and economic impacts of information technology, including impacts on law. For this we need an objective technique of criticism and scientific research about consequences of technology.

\section{THE HISTORY OF INFORMATION PROCESSING}

\section{Some key events}

The history of information processing, information technology, computer science and informatics also furnishes us with striking evidence of the importance of intercultural events and activities, leading to problem solving in the international scientific world as well as in other fields such as the international economy and traffic control.

There are a number of key events, with particular intercultural and interdisciplinary aspects, which gave rise to important technical and social developments in different societies and cultures [3].

Numbers: Number systems and calculation rules were developed from about 2000 B.C. in Babylon (today Iraq) and Egypt to be used in public administration, trade, mathematics, astronomy, calendars, giving rise to exact technics and sciences. 
Scripts with alphabets: At about 1300 B.C. the Phoenicians, living in today's Syria, developed a set of phonetic characters, semantically meaningless (today called an alphabet), for an efficient way of writing. Their motive was the easy documentation of contracts, laws and rules in different languages. This method spread quickly to the ancient Greek and Roman worlds; the effect was reliability in storage and transport of information.

Algorithms: At about 200 B.C. in India, the invention of the decimal point system, using the number 0 , was another key event. This technique was transferred to Arabia in the 9th century. In Bagdad $\mathrm{Al}^{\vee} \mathrm{Hwarizm}$ then developed his "algorithms", highly appreciated in both trade and astronomy. Together with the decimal system, they became popular in commerce and trade first in Italy in the 15th century and finally in all Europe.

Dyadic numbers: In 1679 in Germany, Gottfried W. Leibniz developed the dyadic system for the denotation of numbers, his motives being basically mathematical but also philosophical. Joachim Bouvet, a French Jesuit in Beijing, China, at the court of Emperor Kang Xi, compared the dyadic numbers to the hexagrams, which were written down 5000 years before in China. This caused deep reflection on Leibniz' part also about the religious and philosophical importance of the binary elements, the digits 0 and 1, or the symbols yin and yang, -- -- and ----.

At the same time, the importance of the dyadic system for calculating machines became apparent to Leibniz. He even described a machine, operating with input from this system. The idea of such a machine vanished in history, however, and it was not until 1938 that it was recreated by Konrad Zuse in Berlin, Germany when he constructed the first modern computer working with dyadic numbers.

Calculating machines: Algorithms are symbolic machines [4]. After they had been established, the search started for real machines as processors for algorithms. In 1623, Wilhelm Schickard from Tübingen, Germany, described and had constructed a calculating machine, which mechanised addition and subtraction as well as some steps in multiplication and division. In our context three factors are significant: Schickard's motive was to support astronomical calculations done by Joachim Kepler at this time; he integrated the technique of Napier's bones from England (1617); and he relied on the well-developed art of clockmakers, even calling his machine "Rechenuhr" - calculating clock. His machines were destroyed and forgotten; Schickard's idea was only rediscovered in this century from some of his messages and sketches. 


\section{Integrating information technology into education}

In 1642 Blaise Pascal in Paris, France presented a calculating machine for addition and subtraction, which became well-known in his time and which caused the evolution of more machines. Leibniz was also motivated by Pascal's invention and from 1672 on he worked on concepts for the first machine to perform all four species of calculation completely automatically, which was finished in 1694.

Automata: Another key event can be found in Ancient Greece at about 100 B.C., when Heron of Alexandria invented automata for different control problems, serving religious purposes such as opening and closing temple doors. From our point of view these were programmed machines, as were the "androids", which became popular in Europe in the 17th century. These could perform activities such as playing an instrument or writing with a pen, following a mechanically stored program. These machines were made for entertainment only, although there is some deep reaching philosophical relation to the problem of an artificial human, created with biochemical or technical means.

More pragmatic motives are behind the first attempts to control mechanical weaving machines by punched cards (Falcon 1728, Jacquard 1805 in France) or to mechanise the evaluation of public or commercial statistical data from punched cards (Hollerith 1887, U.S.A.).

Universal machines: Programming and calculating were united in Charles Babbage's design of an Analytical Engine, taking up Jacquard's punched cards, and expected to do all kinds of calculations (Great Britain, 1836).

Thus the idea of a universal computer became more concrete, an idea which had already been anticipated by Leibniz. In today's view he was thinking of a symbolical machine to solve general logical problems, a "calculus rationcinator", universal calculus of thinking. At the same time he discussed a real machine, a "machina ratiocinatrix", which should mechanise the logical processing.

Alan Turing (Great Britain, 1941) wanted to find out which problems are accessible by such a machine. His answer was another symbolic machine, his universal automaton, better known today as the Turing machine. With this he laid the theoretical foundations for the automation of symbolic machines. All our modern computers are models of this concept.

Many more key events like the ones mentioned above can be traced in the history of informatics after Babbage or after Zuse and Mauchly/Eckert, which allow exploitation in education through an informatics model which asks "Where from, where here and now and where to?". 
I cannot go into all of these in this paper. Instead, two examples of subject matter for informatics education shall be presented in some more detail. They may look rather exotic and marginal in the beginning. But looking closer they reveal many elements satisfying subject related intentions as well as general intentions of informatics education.

\section{Hexagrams from the I Ching}

The 64 hexagrams shown in the centre of figure 1, taken from [5], form a complete binary code of length six. Writing 0 for the broken lines (ying) and 1 for the solid lines (yang), from left to right instead from bottom to top as in the hexagrams, you get the system of numbers from 0 to 63 in dyadic (dual) representation.

These hexagrams became well-known through their role in the Chinese 'I Ching', the Book of Changes, where they represent 64 mental states of nature, society or individuals. Changes in their structure by exchanging

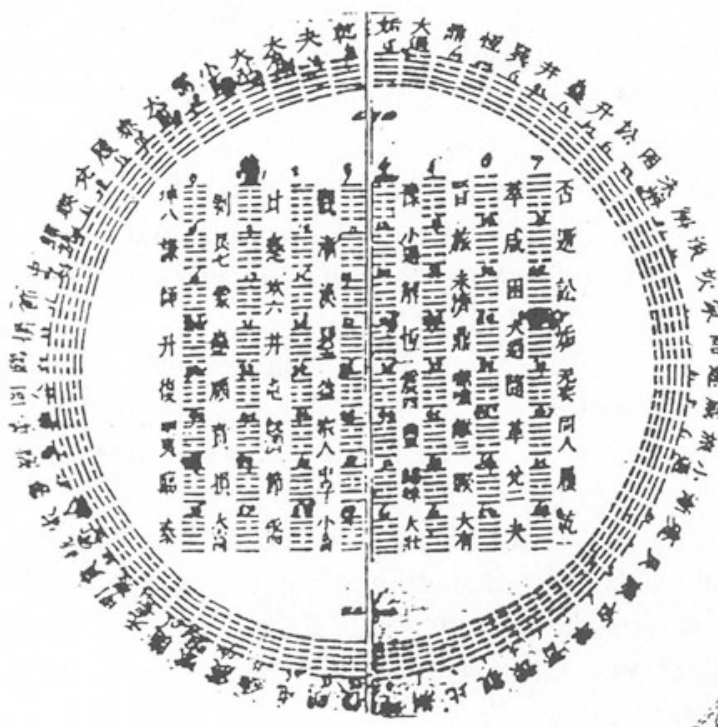
yin and yang lines characterise the eternal transitions between these states. The hexagrams are older than the 'I Ching', at least 4000 years old. They were possibly composed as pairs of the eight trigrams shown in figure 2, taken from [5], which go back to the Chinese sage Fu Hsi.

Figure 1: 64 hexagrams forming a complete binary code [5]

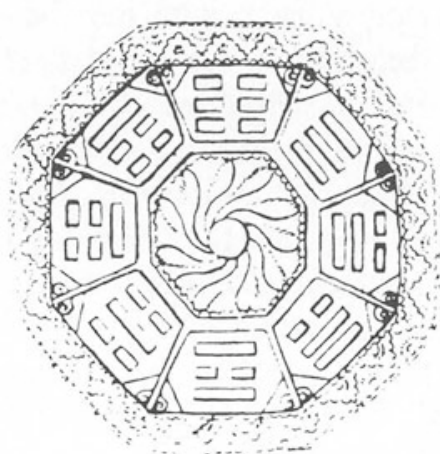

Figure 2: Eight trigrams [5] 


\section{Integrating information technology into education}

Leibniz, who (through figure 1) in 1701 learnt about the hexagrams from Bouvet, a Jesuit at the Court in Beijing, called them "the eldest monument of science". He was fascinated by their relation with the dual numbers which he had just made public. He started a discussion with Bouvet whether the old Chinese had known about the numerical importance of the hexagrams, and if the contemporarians had just forgotten about this.

It is very likely that the old ones did not know. Their intentions with the hexagrams were very different from those of Leibniz. They were looking for a systematic description of the universe. One attempt postulates that at "the very beginning" (Tai Chi), two opposing elementary powers were created, named yang and yin later. These gave rise to four states - the four seasons - and these again to eight trigrams - representing, among other interpretations, eight elementary phenomena from nature, shown in figure 3. This figure is nothing but a binary coding tree. If you expand it in three more steps, you arrive at the 64 hexagrams. Their meanings again are of natural or philosophical character, and so their ordering in the I Ching is different from the one suggested by the binary tree. It is very likely that the ordering in the I Ching refers rather to the changes which can occur from one state to another. At the same time the arrangement of the yins and yangs in each one of the hexagrams has no arithmetic meaning; it stands for the interaction of the yin and yang powers.

The mathematical ingenuity expressed in this binary code is also underlined by the Chinese document from the 12th century, or possibly earlier, shown in figure 4, taken from [6]. Actually it shows a surprising graphical representation of a complete binary tree.

There exists another ordering of the hexagrams, contained in Richard Wilhelm's translation of the I Ching. It has a clearly combinatorial character, starting with the "heaven-hexagram" with six yangs, i. e. zero yins, followed by six hexagrams with exactly one yin, 15 hexagrams with two yins, 20 with three yins and so on.

It is interesting to remark that research done at Rutgers University in New Jersey with American first graders showed that they prefer this kind of ordering when the following problem is put to them: "Here are a lot of blue cubes and red cubes. Please construct all different piles of 3 (or 4, or 5) cubes".

Another proof for the combinatorial skills of the old Chinese scientists is a table from the 10th century, showing the 81 tetragrams, which you gain from three elements, ------, -- -- and -- -- --, for example. 


\section{Chinese calculating machines from the 17th century.}

Some calculating machines were constructed in the Imperial Palace in Beijing at the end of the 17th or early in the 18th century, or they were carried there as gifts from Europe. Two of these machines were rediscovered in 1962, 8 more in 1978. An article about these was published in Chinese in 1980. Two machines were shown in Brussels in 1988. I was able to see three of them in Beijing in 1991. In 1992 Michael

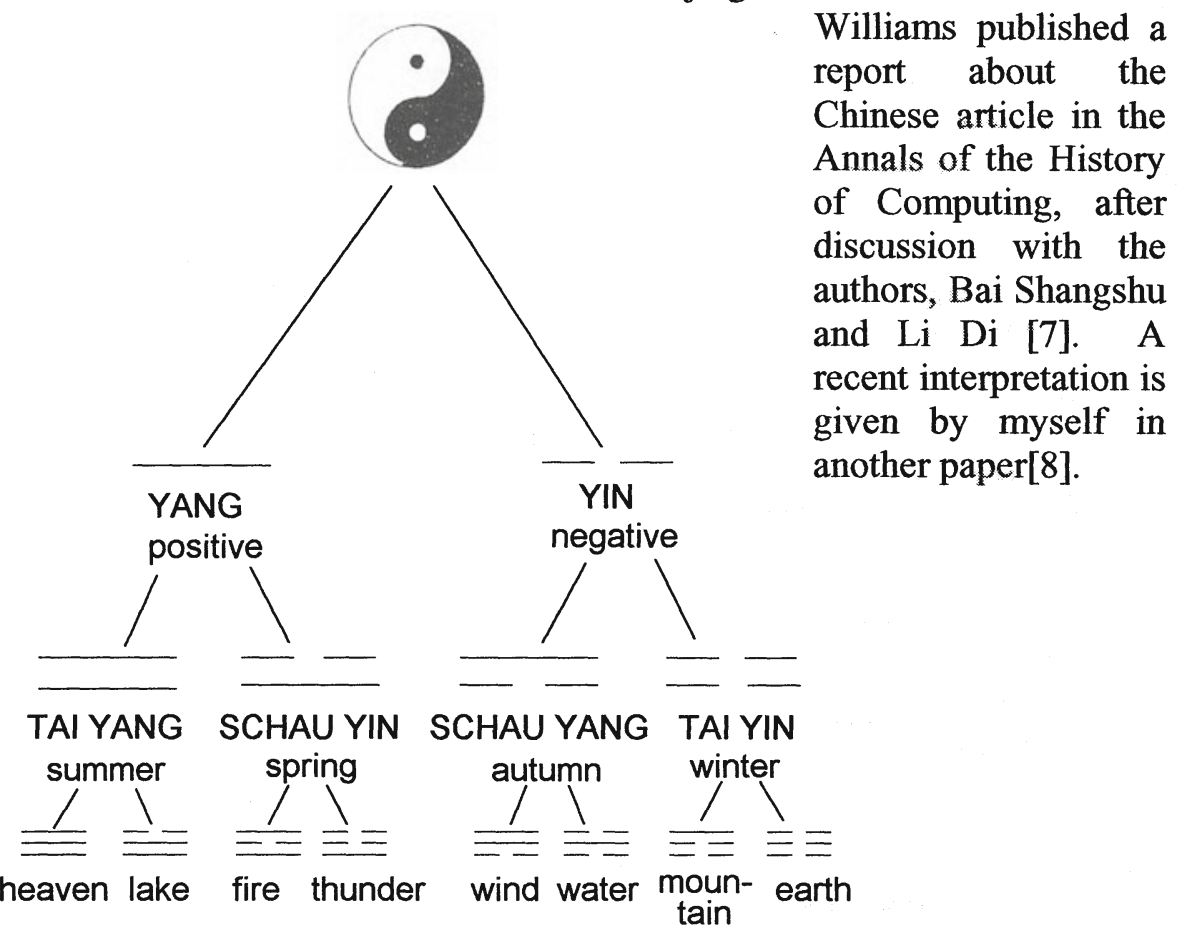

Figure 3: A binary coding tree for for elementary phenomena from Tai Chi

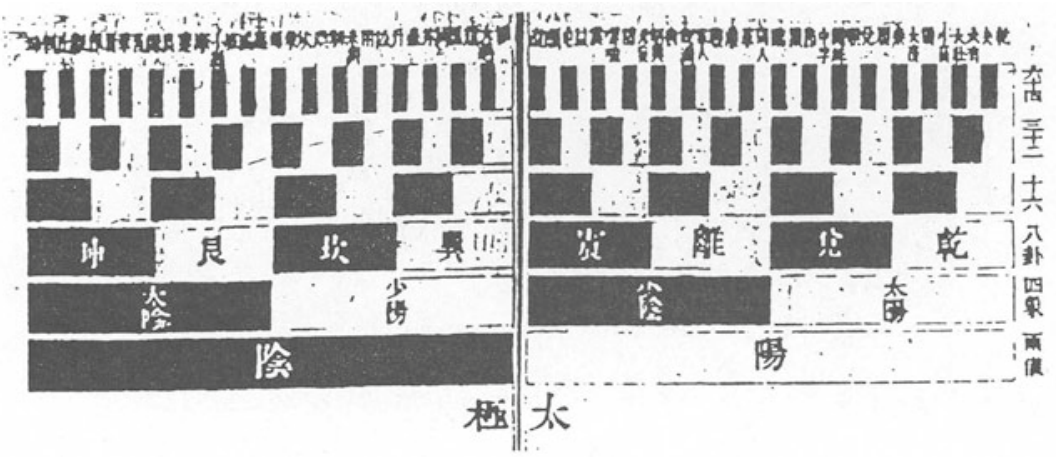

Figure 4: 12 th Century representation of a complete binary tree [6] 


\section{Integrating information technology into education}

Six machines work with decimal tooth-wheels including devices for the carries (figure 5). They allow addition and subtraction. The construction possibly goes back to Christian Huyghens, who developed it after studying Pascal's machine (1643). The technique of the wheel operations is different from Pascal's (figure 6).

Four more devices, supporting multiplications, rely on cylinders each carrying 12 of Napier's bones. They are similar to "calculating cylinders" made by Caspar Schott (1664) and to the multiplication section of Schickard's machine, but they also show impressive improvements by the Chinese constructors.

It is very likely that information about the European devices came to Beijing via the Jesuits, who stayed at the Chinese Court during the reign of Emperor Kang Xi. There exists a letter from Caspar Schott to one of them. It is unknown, however, where exactly the information about the wheel machines came from.

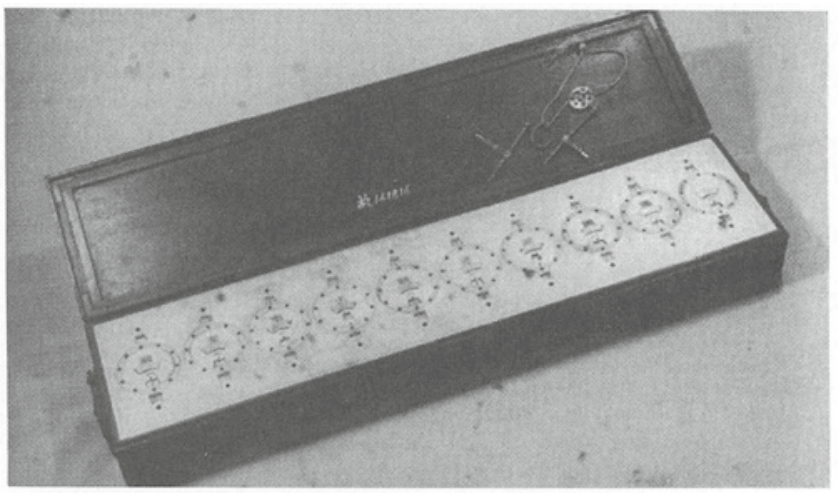

Figure 5: Disc calculating machine with decimal tooth wheels (courtesy of Professor Bai, Beijing)

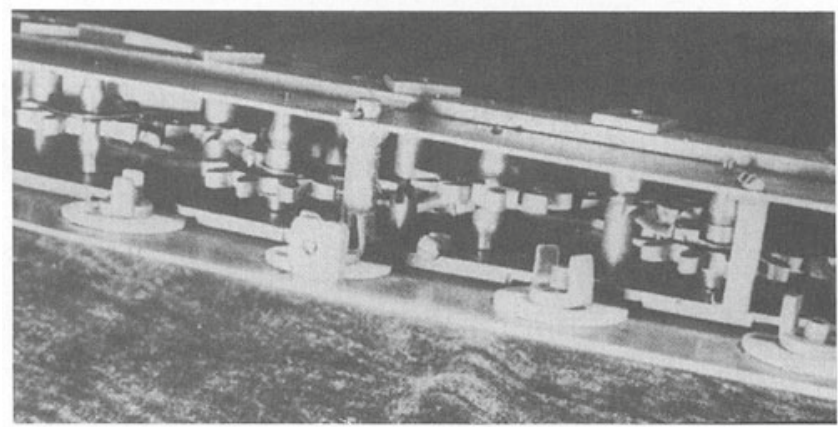

Figure 6: Internal view of disc calculating machine (courtesy of Professor Bai, Beijing) 


\section{CONCLUSIONS}

It is hoped that these two examples illustrate how a teacher can arrange teaching sequences in informatics education which contribute to the development of inter-cultural studies. A positive advantage may be gained for informatics by putting more weight on coding and coding algorithms, for example, instead of on arithmetical problems, or by learning about calculation machines by comparing different historical models. The cross-cultural influences on early developments in informatics is clear, and might be used to substantial effect within informatics education today.

\section{REFERENCES}

1. Ruiz i Tarrago, F. R. (1993) Integration of Information Technology into Secondary Education: Main Issues and Perspectives. In: van Weert (ed.) Guidelines for Good Practice, IFIP Working Group 3.1, Geneva .

2. Graf, K.-D. (1991) Powerful Means in Mathematics and Computer Science Education: Mathematical, Logical, Mechanical and other Roots of Computer Science in History. Journal of the Cultural History of Mathematics 1. Mathematics Education Society of Japan.

3. Rode, H. \& Hansen, K.-H. (1992) Die Erfindung der universellen Maschine, Hannover.

4. Krämer, S. (1988) Symbolische Maschinen, Darmstadt.

5. Zacher, H. J. (1973) Die Hauptschriften zur Dyadik von G. W. Leibniz, Frankfurt am Main.

6. Needham, J. (1954-1965) Science and Civilisation in China, I - IV, (1+2), Cambridge.

7. Bai Shangshu, Li Di \& Williams, R. M. (1992) Chinese Calculators Made During the Kangxi Reign in the Quing Dynasty. IEEE Annals of the History of Computing 14 (4).

8. Graf, K.-D. (1994) Calculating Machines in China and Europe in the 17th Century - The Western View. Brunnstein, K. \& Raubold, G. (eds) 13th World Computer Congress '94 Elsevier, North Holland. 


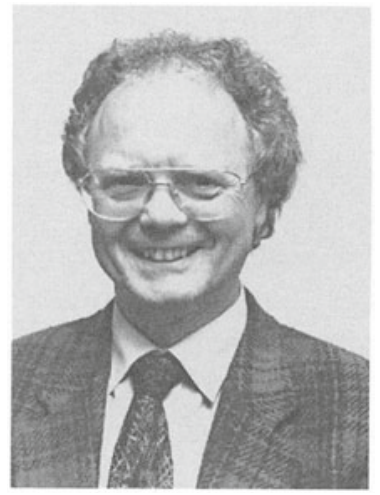

Klaus-D.Graf studied mathematics, physics and education at Chemnitz in Germany and at the University of Illinois in the USA, receiving his $\mathrm{PhD}$ in mathematics in 1966 from the University of Mainz, Germany. He lectured in cybernetics and became a professor of mathematics education in Neuss in 1971, and a professor at Freie Universität, Berlin in 1975. From 1980 he has specialised in informatics education, computer use in mathematics and CAL. He was a visiting professor in the Republic of China in 1987 and has authored and co-authored textbooks on computer science and information technology in schools. 